\title{
Investigating Stakeholders' Perceptions of Feasibility and Implications of Modular Construction-Based Post-Disaster Reconstruction
}

\author{
Pedram Ghannad ${ }^{1}$, Yong-Cheol Lee ${ }^{2 *}$, and Jin Ouk Choi ${ }^{3}$ \\ ${ }^{1}$ Ph.D. Student, Bert S. Turner Department of Construction Management, Louisiana State \\ University \\ ${ }^{2}$ Assistant Professor, Bert S. Turner Department of Construction Management, Louisiana State \\ University \\ ${ }^{3}$ Assistant Professor, Department of Civil and Environmental Engineering and Construction, \\ University of Nevada, Las Vegas \\ *Corresponding author's e-mail: yclee@lsu.edu
}

\begin{abstract}
Natural Disasters cause major adverse social and financial effects by destroying homes and infrastructures. For example, Hurricane Katrina in August 2005 damaged over 214,700 homes in New Orleans and forced over 800,000 citizens to live outside of their homes due to flooding. Thus, these disasters require a quick and efficient response to post-disaster housing issues and provide resources for temporary houses for short-term disaster relief and reconstruction of destroyed and damaged housing for full rehabilitation. Reconstruction of permanent housing for disaster victims is one of the most time-consuming activities in the post-disaster recovery process. However, time is a critical factor which should be minimized for the restoration of affected communities. Modularized construction is a promising solution for improving the process of post-disaster housing reconstruction because of its inherent characteristic of time-efficiency. This paper aimed to evaluate prefabricated modular construction potentials as an approach that can facilitate the design and construction phase of post-disaster reconstruction. An extensive literature review has been carried out to identify the features of modularized construction which can add value to the post-disaster recovery process. To investigate the suitability and feasibility of implementing modular construction for post-disaster reconstruction and also identify major barriers of its implementation, a survey has been conducted in 2018 among AEC experts who were experienced in the prefabricated construction industry and/or involved in post-disaster reconstruction projects. The results of the study indicate that prefabricated modular construction is a promising approach to improve time-efficiency of post-disaster reconstruction and tackle challenges of current practices by its unique benefits such as reduced demand for on-site labor (overcome local labor pool constraints impacted by the disaster) and resources (overcome shortage of equipment and materials), shorter schedule (due to concurrent \& non-seasonal), reduced site congestion, and improved labor productivity (due to assembly line-like and controlled environment).
\end{abstract}

\section{KEYWORDS}

Post-disaster Reconstruction; Modular Construction; Prefabrication; Modularization 


\section{INTRODUCTION}

The disaster has been defined and investigated from various perspectives by several authors. Smith et al. (2014) defined the disaster as an unexpected natural or manmade phenomenon which causes a large number of lives and property losses. Forcael et al. (2014) claimed that 373 natural disasters occurred which caused about 296,000 lives losses and about 110 million dollars cost for about 208 million people directly or indirectly. FEMA categorizes disaster as the following four phases: (1) mitigation; (2) preparedness; (3) response; and (4) recovery (FEMA 2005). Among these phases, this study aims to improve the recovery phase, which requires an enormous amount of time, fund, and effort after a disaster event.

From the construction management perspective, the rapid recovery of damaged houses and infrastructures is one of the urgent challenges. The most critical and time-consuming step of a post-disaster recovery and reconstruction process is promptly providing permanent housing for affected people. This process can take up to five years (FEMA, 2005) or even more, up to 10 years (Goodyear, 2014), according to the severity of the disaster. Undoubtedly, it is a very long period of time for communities and people to restore their normal livelihood. Therefore, time is a key factor in the reconstruction process to minimize the impacts on the communities. To explore a new approach to tackling the challenge of time in the post-disaster recovery, this study investigated a modular construction approach for mass reconstruction. Time-efficiency is an inherent characteristic of modular construction, which offers great potential for it to be a desirable strategy for post-disaster housing reconstruction. In this paper, the authors investigated the feasibility and perception of modular construction-based post-disaster reconstruction with the survey from industry professionals and identified the competitive features of modular construction for facilitating the disaster recovery process.

\section{METHODOLOGY}

To achieve the objectives of this study, a comprehensive literature review has been conducted to identify the major challenges of a post-disaster reconstruction process. Modular construction also has been investigated by reviewing the literature to find out the features and capabilities that can be leveraged to improve the current status of a post-disaster reconstruction process. The results have been validated by subject matter experts who have been involved in relevant projects with a diverse background. According to the extensive literature review, an online questionnaire has been designed and distributed among experts. To verify the findings, an analytical comparison has been done between the results of the literature review and the survey.

\section{LITERATURE REVIEW}

\section{Post-disaster recovery and reconstruction process}

This study divides the following two parts of post-disaster reconstruction: housing and infrastructures (e.g. roads, electricity, ports, etc.). Both are the urgent issues, but housing reconstruction after a disaster causing a large number of victims has been mostly addressed in diverse studies. Two types of dwelling have been determined by the terms "shelter" and "housing" (FEMA, 2005). The shelter is a temporary accommodation provided until the victims can relocate to permanent houses. On the other hand, "Housing" refers to permanent dwelling with all 
requirements including physical, social, and administrative infrastructure (Gunawardena, 2014). In other words, shelters are provided as the short-term solution of relief process and permanent houses must be provided in the long run to recover the normal livelihood of the disaster-affected communities.

The post-disaster housing reconstruction process encounters similar challenges to other housing projects in addition to several other challenges due to their special situation. These added challenges have been discussed in literature extensively as shown in the following key factors.

(1) Time: Disaster recovery projects should be completed as quick as possible to minimize impacts on affected victims. As mentioned above, the completion of such projects may take at least 5 years which is a considerably long time period for restoration of affected people. Tas et al (2010) stated that 'time' is the most critical factor that determines the post-disaster permanent housing strategy. In addition to the reduction in recovery time, the time is crucial to reduce construction costs supported by federal government or personal resources. Weerakoon et al. (2007) studied the post-disaster recovery process of Indian Ocean Tsunami in Sri Lanka and found that wages of labor and price of material considerably increased (about 15-25 percent) over the recovery time due to inflation.

(2) Funding: One of the most common challenges of the post-disaster recovery process is funding for reconstruction, which can be worsened by deploying inappropriate strategies. Sometimes, spending excessive fund in the relief process such as building shelters may cause inadequacy of funds for housing reconstruction projects (Lloyd-Jones, 2006). Availability of adequate fund is necessary for starting a housing reconstruction process. This fund is mostly from insurance or aids from donors and governments. The challenge of funding is more significant when the insurance coverage is not available so that starting the reconstruction process highly depends on aids from outside (Hidayat and Egbu, 2010). Due to a large number of damages and limited funds, communities affected by a disaster always have difficulties to properly and promptly initiate their recovery process with the insufficient fund for reconstruction. The other challenge regarding funding resides in allocating given funding efficiently and steadily to fulfill the projects' needs, which require wise and systematic portfolio management.

(3) Resources and Materials: The other common bottleneck of housing reconstruction projects is the shortage of required resources and construction materials. Housing and infrastructure recovery projects supposed to be simultaneously started by the public and private sections require an enormous amount of construction resources and materials. Since a natural disaster event sweeps out local production facilities and supply systems and causes disruption of transportation systems, it frequently brings serious problems in procuring construction resources in a timely manner during the recovery process (Chang et al., 2010). Several studies revealed that the shortage of materials leads to adversely affect the projects' objectives such as project suspension, quality defects, cost overrun, and delivery delay (Boen, 2006; Steinberg, 2007). In addition, Tabmbe et al. (2018) stated that during post-earthquake housing reconstruction in the Sikkim Himalaya, many houses construction processes were suspended because the supply of stock material was not able to fulfill the rising demand. 
(4) Workforce: Several case studies revealed that inadequacy of available skilled workforce during construction projects is one of the decisive issues that negatively influence the post-disaster reconstruction projects. The findings of the Koria's study (2009) on the reconstruction projects in Sri Lanka indicated that the lack of field staff's relevant experience or training to handle a large and complex project was a key issue in delaying the recovery process. Arshad \& Athar (2013) also stated that locals assisting the recovery projects have had a lack of knowledge and/or skills in Pakistan after the 2005 Earthquake. Inadequate worker's skill may lead to poor quality of the constructed facility.

(5) Planning, Communication, and Coordination: The other challenges are ineffective coordination and communication among agencies involved in reconstruction. Involvement of federal and local government, various funding agencies, donors, and other stakeholders requires a comprehensive approach for systematic coordination and extensive knowledge of planning. Roosli et al (2012) identified a lack of expertise and knowledge in the relevant authorities acting as a major impediment in the housing reconstruction process.

(6) Resiliency and Sustainability: The post-disaster period has been recognized as an opportunity to improve quality, resiliency, and sustainability and reduce vulnerability to future disasters (Davidson et al, 2006). Thus, to establish new facilities and communities, practitioners need to consider their resiliency and sustainability to prepare any unexpected future events. In addition, there are several factors that must be considered in the post-disaster reconstruction process including limitation of transportation and accessibility of affected areas.

\section{Modular construction and prefabricated houses}

Modular construction is a modern construction methodology that was introduced as an alternative for traditional cast-in-situ (stick-build) construction. In recent years, modular construction has attracted immense attention from many countries because of its inherent superiority of this technology including, but not limited to, construction waste reduction (MBI, 2010), improved quality control (Judy, 2012)), noise and dust reduction (Pons \& Wadel, 2011), higher standards for health and safety (CII, 2002; CII, 2011; MBI, 2010), time efficiency (CII, 1987; CII, 2002; MBI, 2010), cost saving (Lawsen et al., 2012), reduced labor demand (Nadim \& Goulding, 2010), and low resource depletion (Won et al., 2013). Choi (2014) provided a comprehensive summary of the advantages and disadvantages of modularization in previous studies. The inherent characteristics of modular construction make it the most suitable method for post-disaster housing reconstruction. In the following section, the identified features of modular construction which can promisingly tackle the mentioned challenges of post-disaster recovery process will be analyzed in detail.

\section{(1) Time-efficiency}

One of the most important characteristics of modular construction, which make it a fitting approach for post-disaster housing reconstruction, is the time-efficiency. According to Lawson et al. (2012), it can decrease project completion time about 50\% in comparison with traditional on-site construction. The simultaneous process of mass production and on-site installing of modules will shorten the construction time. This time saving will benefit the recovery process by both minimizing the restoration time for affected communities and avoiding labor and 
material cost fluctuations. Furthermore, the flexibility of the modular construction method offers great potential for saving time. All components of a building can be configured as a module together or separately. Shapes and sizes of the modules can vary to comply with the limitations of transportation and technical aspects of construction, such as truck dimensions and height or weight restrictions.

\section{(2) Long-term cost benefits}

Although it seems that modular construction requires more initial cost and investment, the long-term benefits of this approach are greater in comparison with traditional construction. Rogan et al. (2000) assess the costs and benefits of modular construction as against traditional construction, for a typical four-story residential building in London. Where the initial investments have only been a mere 2 percent higher for modular construction, but it has received greater benefits, from the beginning of the life of the structure. With a 39 percent greater turnover estimated and a 43 percent higher Internal Rate of Return (IRR), modular construction was clearly shown to provide more benefits to the builder as well as the client. Modular construction has also this potential to reduce the material delivery cost, accommodation cost of labors, and on-site usage of equipment such as crane (Fagerlund 2001).

\section{(3) Reduced demand for labor and resources on-site}

As modular construction exports site-based works to off-site, 1) on-site labor demand is reduced which leads to reduced onsite accommodation costs (Fagerlund 2001; Gotlieb et al. 2001); 2) resources demand on the job site can be also minimized. This helps the fabricator to hire skilled labor in a fabrication shop and the contractor to hire skilled labor on the job site more selectively with lower costs (Choi 2014). A high percentage of the construction process of a modular structure is a pre-planned process carried out in a factory environment. External parties would only get involved in the on-site construction. Modular units are generally built with provisions for services. It is only a matter of connecting them on-site once the modules are assembled. Furthermore, since the interiors, as well as façades, roofs, etc., are all preconstructed into the modules, the required planning becomes much simpler. Modular construction potentially has the ability to provide a more workable platform for institutions of various disciplines, such as contractors, governmental institutions, non-governmental organizations, and humanitarian agencies, to work together and produce better results; however, this is the desired, not the current practice in the off-site construction industry

\section{(4) Resilient and sustainable houses}

Modular construction helps to improve quality control (Lapp and Golay 1997) and the production of a housing module is done in a highly controlled environment. The quality checks inside a mass production facility will be more reliable compared to on-site construction, especially in a post-disaster scenario where on-site construction will be under heavy pressure for delivery. This will make sure that the final product suits them with respect to structural stability, livability, and sustainability.

The extensive conducted literature review indicated that modular construction with its inherent characteristics is a promising strategy for rapid post-disaster housing reconstruction. The in-detail analysis of previous case studies also revealed that modular construction can bring the potential to improve the post-disaster recovery process and address most of its challenges. Table 1 briefly 
describes the finding of the literature review. According to the key factors of modular construction-based post-disaster recovery from this literature review, the authors designed and conducted a survey to collect the industry experts' opinions with the purpose to validate our findings and investigate the feasibility and suitability of modular construction for facilitating postdisaster housing reconstruction.

Table1. A brief description of the literature review

\begin{tabular}{lll}
\hline $\begin{array}{c}\text { Challenges of post-disaster } \\
\text { recovery projects }\end{array}$ & \multicolumn{1}{c}{$\begin{array}{c}\text { Characteristics of modular } \\
\text { construction }\end{array}$} & $\begin{array}{c}\text { Ability to solve the } \\
\text { issue }\end{array}$ \\
\hline Time & Time-efficiency & HIGH \\
Funding & Long-term benefits & LOW \\
Quality & High-quality controlled environment & HIGH \\
Planning & Pre-planned process & MEDIUM \\
Resource availability & Resource integration & MEDIUM \\
Skilled workforce & Minimum required on-site skill & HIGH \\
\hline
\end{tabular}

\section{VALIDATION BY A SURVEY OF EXPERTS}

In order to validate the findings of our literature review, 18 industry experts were invited to participate in a short survey. The survey contains questions about the feasibility of utilizing modular construction for the reconstruction of the individual building as well as a mass reconstruction of houses with different designs. The invited experts have also been asked to identify the benefits and the barriers of modular construction implementation in the post-disaster recovery process by choosing options from one (1) to four (4); one being strongly disagreed or no importance and four being strongly agreed or high importance. The survey found that the participants have 375+ years of experience in the AEC/FM industry. 94.4 participants (17 out of 18) have been involved in at least one modular construction in the last five years. In addition, $38.9 \%$ of them ( 7 out of 18 ) have been involved in a post-disaster reconstruction project using modular construction. The Cronbach's $\alpha$ (alpha) (Cronbach, 1951) has been calculated for testing the consistency of responses. The alpha value is equal to 0.845 which indicates the "Good" consistency (Cortina, 1993) in our responses.

\section{Findings of the survey and discussion}

Literature provided several pieces of evidence that the implementation of modular construction can efficiently address the challenges during the post-disaster reconstruction process. The result of the survey also supports the benefits identified in the literature review about the suitability of a modular approach for post-disaster recovery by the average point of 3.16 (out of 4.00) for individual houses and 3.00 for mass construction with different designs. This question has also been added to another survey about modular construction designed and distributed by our collaborators. The results of the survey are also consistent with the literature because over 90 percent of survey participants (40 out of 44) responded that modular construction/prefabrication can be used for mass post-disaster reconstruction of facilities (average point of 3.23 out of 4). The respondents believed that the process of modular construction is "quicker", and this means 
"quicker relief". They stated that prefabricators can work in non-affected areas simultaneously with clean-up in affected areas so that they can deliver their products as soon as possible. However, there were few comments from negative respondents. One believed that it can be only the shortterm solution. It has also asserted that amount of preconstruction planning and coordination can impede the quick response to disaster even with modular construction.

Most of the respondents believe that modular construction can reduce the time of post-disaster reconstruction projects and it received point of 3.27, while cost reduction has a lower point (2.88) and consequently lower support among the experts. The experts also evaluated the benefits of modular construction and their influence on making this approach suitable for post-disaster reconstruction. The results have been shown in Figure 1. The most important characteristics that benefit the post-disaster recovery process are as follows:

- Easier access to required labor and material

- Improved Schedule

- The potential for a quick response

- Better Predictability/Reliability

- Increased Productivity

- Sufficient Labor Supply

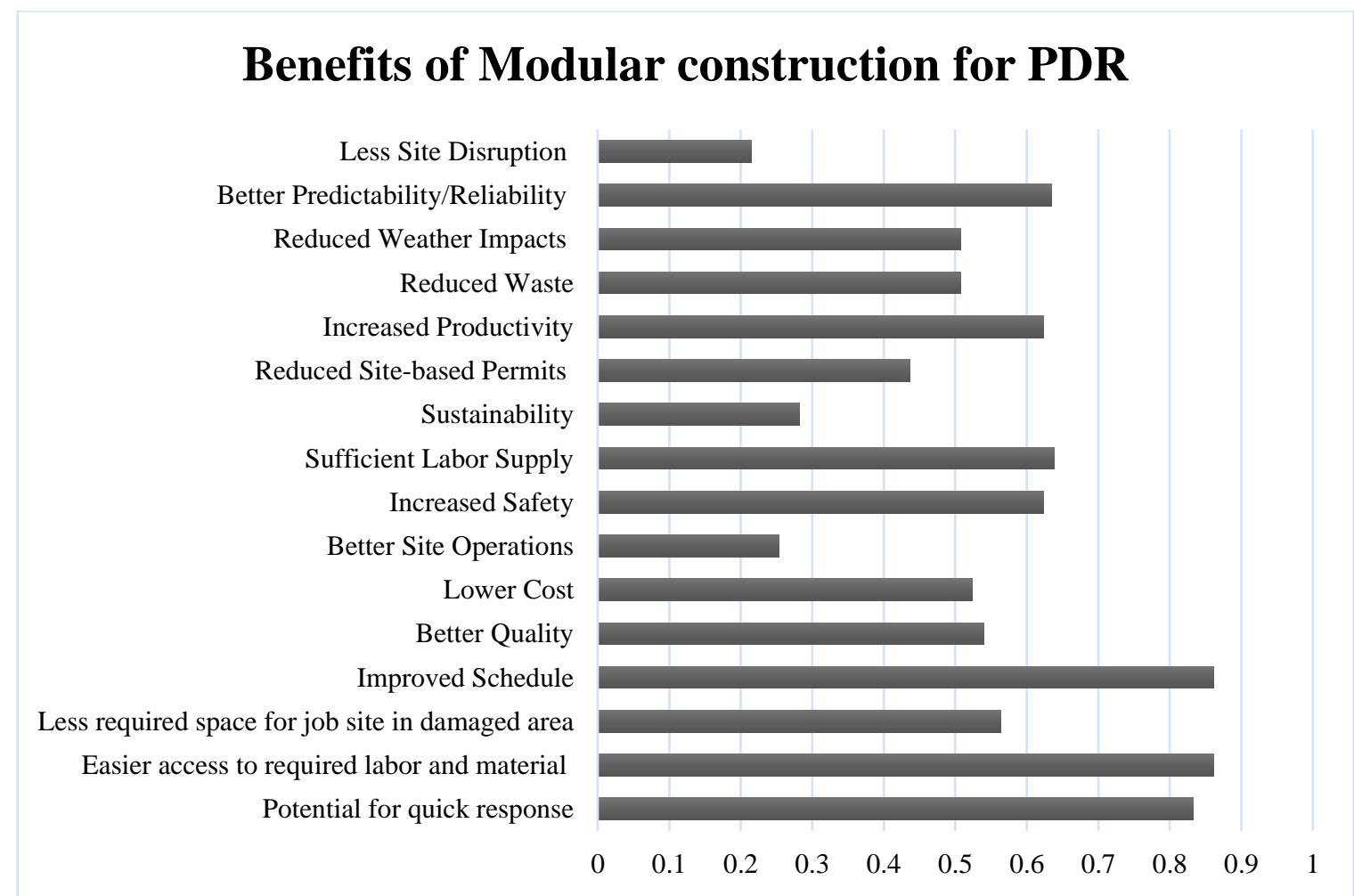

Figure 1. Benefits of modular construction and their importance in its suitability for post-disaster recovery

These factors are mostly related to the time-efficiency of modular-based construction, minimum requirement of skill and labor, and pre-planning features of modular construction, which reinforce 
the finding of our literature review. However, the authors expected stronger support for the ability of modular construction in time reduction of post-disaster recovery projects. It indicates that although modular construction can offer the most time-efficient strategy for reconstruction, a construction method is not the only factor that determines the completion time of the projects because of a complex environment and situation after a disaster. The chaotic environment, social and political considerations, funding limitation is the other factor that can affect the completion time for post-disaster recovery projects. Moreover, it should be noted that in contrast with the literature that indicated modular construction can improve the quality of construction in housing reconstruction projects due to its potential for implementing quality control procedures, the respondent did not identify the quality as an effective factor for post-disaster reconstruction projects. This conflict might be because of overlooking of quality during the post-disaster reconstruction. Importance of time, pressure from stakeholders for projects completion, and high demands are the factors that lead to scarifying the quality to obtain the other objectives of the project. Tabmbe et al. (2018) briefly indicated that low-quality of prefabricated houses is one of the shortcomings of this strategy for post-disaster permanent housing reconstruction.

As a part of the survey, participants evaluated the barriers that impede employing modular construction as the main strategy for post-disaster reconstruction. The results show that they identified design and construction culture, quality concerns, and unpredictable conditions after a disaster as the most important barriers. Design and construction culture barrier for modular construction is not limited to post-disaster reconstruction projects. Gan et al. (2018) found that "protectionism" and "conservatism" inherent within the AEC industry culture play a pivotal role in limiting technological innovations such as modular construction. Regarding the quality concerns, it is obvious that the satisfaction of end users is always a key concern. Although the affected individuals may eventually be thankful for the resettlement of their livelihoods after possibly losing all their possessions, it must be understood that they are entitled to be opinionated of the quality of the finished product. For this reason, institutions such as FEMA (2005), APEC (2009) and UNDRO (1979) have set standards for post-disaster housing reconstruction. However, high demands after a disaster might lead to several deficiencies in module production and undesired defects in the final products. So, more reliable quality control procedures are needed to fulfill most of the requirements.

\section{CONCLUSION}

It is observed that time is a critical factor in the reconstruction of permanent housing for disaster victims and it should be minimized for the restoration of affected communities. Modular construction can drastically improve this time gap. The faster construction times, which are characteristic of modular construction, make this form of construction a great solution for providing faster permanent houses. Factors such as scarcity of resources, deficiencies in transportation, funding, etc. can still have a detrimental effect on the efficiency of modular construction. However, such factors can be expected in a post-disaster scenario and must be planned. A great advantage of using modular structures as a post-disaster housing solution is that much of the expertise in reconstruction is directed to one solution provider. This feature makes stakeholders able to address common challenges of the post-disaster reconstruction process, lack of skilled workforce and inefficiencies in planning, communication, and coordination. 
MOC SUMMIT / MAY 2019

\section{REFERENCES}

APEC (Asia Pacific Economic Cooperation) (2009). Guidelines and Best Practices for PostDisaster Damage and Loss Assessment; Report from APEC Workshop on Damage Assessment Techniques. Hong Kong, Corporate Social Responsibility in Asia.

Arshad, S., \& Athar, S. (2013). Rural housing reconstruction program post-2005 earthquake: Learning from the Pakistan Experience: A manual for post-disaster housing program managers. World Bank.

Chang, Y., Wilkinson, S., Potangaroa, R., \& Seville, E. (2011). Identifying factors affecting resource availability for post-disaster reconstruction: a case study in China. Construction Management and Economics, 29(1), 37-48.

Chang, Y., Wilkinson, S., Potangaroa, R., \& Seville, E. (2010). Resourcing challenges for postdisaster housing reconstruction: a comparative analysis. Building Research \& Information, 38(3), 247-264.

Choi, J. O. (2014). Links between modularization critical success factors and project performance (Doctoral dissertation).

CII (1987). "Constructability improvement using prefabrication, preassembly, and modularization ", C. B. Tatum, J. A. Vanegas, and J. M. Williams, eds., The University of Texas at Austin: Construction Industry Institute, Austin, TX.

CII (2002). "Implementing the Prefabrication, Preassembly, Modularization, and Offsite Fabrication Decision Framework: Guide and Tool." The University of Texas at Austin: Construction Industry Institute, Austin, TX.

CII (2012). "Industrial Modularization: How to Optimize; How to Maximize." The University of Texas at Austin: Construction Industry Institute, Austin, TX.

Cortina, J. M. (1993). What is coefficient alpha? An examination of theory and applications. Journal of applied psychology, 78(1), 98.

Cronbach, L. J. (1951). Coefficient alpha and the internal structure of tests. psychometrika, 16(3), 297-334.

Davidson, C. H., Johnson, C., Lizarralde, G., Dikmen, N., \& Sliwinski, A. (2007). Truths and myths about community participation in post-disaster housing projects. Habitat international, 31(1), 100-115.

Fagerlund, W. R. (2001). Decision framework for prefabrication, pre-assembly and modularization in industrial construction (Doctoral dissertation, University of Texas at Austin).

FEMA. (2005). Planning for Post Disaster Recovery and Reconstruction, Washington D.C., FEMA (Federal Emergency Management Agency).

Forcael, E., González, V., Orozco, F., Vargas, S., Pantoja, A., \& Moscoso, P. (2014). Ant colony optimization model for tsunamis evacuation routes. Computer-Aided Civil and Infrastructure Engineering, 29(10), 723-737.

Goodyear, R. (2014). Housing in greater Christchurch after the earthquakes: Trends in housing from the Census of Population and Dwellings 1991-2013. Wellington, New Zealand: Statistics NZ.

Gotlieb, J., Stringfellow, T., and Rice, R. (2001). "Power Plant Design Taking Full Advantage of Modularization." Power Engineering, 31.

Gunawardena, T., Tuan, N., Mendis, P., Aye, L., \& Crawford, R. (2014). Time-Efficient PostDisaster Housing Reconstruction with Prefabricated Modular Structures. 
Hidayat, B., \& Egbu, C. O. (2010, September). A literature review of the role of project management in post-disaster reconstruction. In Procs 26th Annual ARCOM Conference (pp. 1269-1278). Association of Researchers in Construction Management.

Koria, M. (2009). Managing for innovation in large and complex recovery programmes: Tsunami lessons from Sri Lanka. International Journal of Project Management, 27(2), 123-130.

Lapp, C. W., \& Golay, M. W. (1997). Modular design and construction techniques for nuclear power plants. Nuclear Engineering and Design, 172(3), 327-349.

Lawson, R. M., Ogden, R. G., \& Bergin, R. (2011). Application of modular construction in highrise buildings. Journal of architectural engineering, 18(2), 148-154.

Lloyd-Jones, T. (2006). Mind the Gap! Post-disaster reconstruction and the transition from humanitarian relief. RICS.

MBI (2010). "Improving Construction Efficiency \& Productivity with Modular Construction." Modular Building Institute, VA.

McIntosh, J. (2013). The implications of post disaster recovery for affordable housing. In Approaches to Disaster Management-Examining the Implications of Hazards, Emergencies and Disasters. InTech.

Nadim, W., \& Goulding, J. S. (2010). Offsite production in the UK: the way forward? A UK construction industry perspective. Construction innovation, 10(2), 181-202.

Oxfam (2003). Guidelines for Post Disaster Housing, Version 1. Oxford, Oxfam.

Rogan, A. L., Lawson, R. M., \& Bates-Brkljac, N. (2000). Value and benefits assessment of modular construction. Steel Construction Institute, Ascot, UK.

Roosli, R., Vebry, M., Othuman Mydin, M. A., \& Ismail, M. (2012). BUILDING AND PLANNING OF POST-DISASTER REHABILITATION AND RECONSTRUCTION. International Journal of Academic Research, 4(1).

Tambe, S., Pradhan, S., Donka, P., \& Singh, P. (2018). Post-earthquake housing reconstruction in the Sikkim Himalaya: approaches, challenges, and lessons learnt. Development in Practice, 28(5), 647-660.

Tas, N., Tas, M., \& Cosgun, N. (2011). Permanent housing production process after 17 August 1999 Marmara Earthquake in Turkey. International Journal of Strategic Property Management, 15(3), 312-328.

Tas, M., Tas, N., \& Cosgun, N. (2010). Study on permanent housing production after 1999 earthquake in Kocaeli (Turkey). Disaster Prevention and Management: An International Journal, 19(1), 6-19.

UNDRO (1979). Natural Disasters and Vulnerability Analysis. Geneva, UNDRO (United Nations Disaster Relief Organization).

Weerakoon, D., Jayasuriya, S., Arunatilake, N., \& Steele, P. (2007). Economic challenges of posttsunami reconstruction in Sri Lanka (No. 75). ADB Institute Discussion Papers.

Won, I., Na, Y., Kim, J. T., \& Kim, S. (2013). Energy-efficient algorithms of the steam curing for the in situ production of precast concrete members. Energy and buildings, 64, 275-284. 